\title{
COVID-19 and Obesity-the Management of Pre- and Post-bariatric Patients Amidst the COVID-19 Pandemic
}

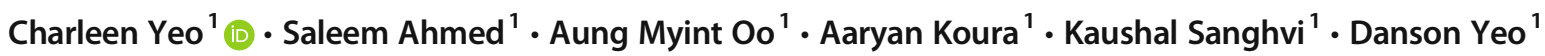 \\ Published online: 9 May 2020 \\ (C) Springer Science+Business Media, LLC, part of Springer Nature 2020
}

\section{Introduction}

The coronavirus disease 2019 (COVID-19) caused by the severe acute respiratory syndrome coronavirus 2 (SARS-CoV2) was declared a global pandemic by the World Health Organization (WHO) on 11 March 2020. Since then, countries and hospitals worldwide have been diverting precious healthcare resources towards the management of COVID-19 patients.

Bariatric surgery is currently an effective treatment modality for obesity and associated metabolic syndrome [1,2]. However, in this unprecedented global crisis, it is important that nonurgent clinic appointments and operations be postponed in order to conserve resources and reduce risks of viral transmission [3]. Treatment for chronic diseases such as obesity and metabolic syndrome has been postponed, and elective surgeries such as bariatric operations have been suspended [4].

Despite the ongoing pandemic, patients with chronic conditions such as obesity and metabolic syndrome should not be neglected. Similarly, both pre- and post-bariatric surgery patients should also not be ignored. Many post-bariatric surgery patients, especially those post-Roux-en-Y gastric bypass (RYGB), may have nutritional deficiencies that require close follow-up [5]. Additionally, diabetic patients will also require monitoring to ensure adequate glycemic control during this period.

We outline the impact of COVID-19 on obesity and its associated metabolic syndrome, and the measures that can be taken to ensure that our patients continue to receive adequate care during this pandemic.

Charleen Yeo

charleen.yeo@gmail.com

1 Department of Upper Gastrointestinal and Bariatric and Metabolic Surgery, Tan Tock Seng Hospital, Singapore, Singapore

\section{Impact of COVID-19 on Obesity and Metabolic Syndrome}

Due to the alarming increase in COVID-19 cases and widespread community transmission, many countries have declared a state of emergency, and cities have gone into lockdown. Non-essential travel and work have been halted, which has affected millions of people. Lockdowns may create stressful situations for people who remain cooped up at home for long hours, which can potentially result in increased food consumption due to boredom or even stress eating behavior [6, 7]. To avoid going out of the house, people also tend to stock up on and consume preserved and canned food, which are known to be high in fat, calories, and sodium content [8].

With most public recreational facilities such as gyms, swimming pools, tennis/basketball courts et cetera closed, options for exercise are also limited. Exercise activities have been largely limited to walking/running, which may not be suitable for patients with higher grades of obesity with associated joint pains or arthritis. Ongoing dedicated weight management programs encompassing of group therapy sessions, allied health visits, and endocrinologist reviews have all been halted.

Studies have also shown that COVID-19 patients with obesity and other comorbidities including metabolic syndrome are at a higher risk of requiring intensive care unit (ICU) stay and have increased rates of mortality [9, 10]. Obesity is associated with compromised pulmonary function (decreased expiratory reserve volume, functional capacity, and lung compliance) and an increase in inflammatory cytokines which may increase the morbidity of COVID-19 infections [11]. The association between DM and increased lung ACE2 enzyme expression may also suggest increased severity of COVID-19 infection [10]. Hence, it is imperative not to neglect this important subset of patients. 


\section{Managing Obese and Pre-bariatric Patients}

This group of patients should receive timely reminders and education about the various options of healthy eating. Flyers/pamphlets detailing different methods of healthy home cooking can be distributed. In certain patients with class II/III obesity, meal replacements may also be an alternative. Exercise should also be encouraged - even simple exercises including brisk walking. As most countries are under a lockdown, it is important to develop in-house exercise schedules consisting of activities such as static cycling, yoga, and tai-chi, which have been shown to improve cardiovascular risk factors [12]. Maintaining a healthy lifestyle with adequate physical activity has been proven to have a positive impact on our immune system [13].

With the advent of technology, patients can also utilize various online platforms to guide their weight and comorbidity management. Online social support group gatherings and exercise classes can be initiated for patients who are under lockdown. Electronic applications such as meal/caloric trackers and hypocount trackers can also be utilized.

\section{Managing Post-bariatric Patients}

In our institution, post-bariatric surgery patients are followed up closely during their first post-operative year. These followups are conducted by a multidisciplinary team including surgeons, endocrinologists, psychologists, dieticians, and physiotherapists. The aim of these regular visits is fourfold: to manage their other comorbidities, nutritional monitoring, assessing for surgical complications, and to monitor their weight loss response and/or any weight regain. Bariatric patients, especially those post-RYGB, are at risk of both macroand micro-nutritional deficiencies such as iron, folate, vitamin B12, and vitamin D $[14,15]$. If undiagnosed and untreated, such long-term nutritional deficiencies can lead to multiple medical problems like anemia and osteoporosis. The postoperative recovery for bariatric patients may also have an impact on their psychosocial well-being, eating behavior, and social functioning $[16,17]$. Studies have shown that feelings of "abandonment" and "isolation" may dominate patients' recovery experience [16]. It is essential to ensure that our post-surgical patients remain psychologically and socially supported. In the long run, concerns of DM relapse [18], inadequate weight loss, or weight regain also need to be addressed [19]. Studies have shown that elevated levels of distress secondary to COVID-19 can lead to emotional dysregulation, leading to increased impulsivity and binge eating symptoms before and after bariatric surgery [20,21].

We propose the following measures to ensure that our postbariatric surgery patients still receive adequate support and follow-up during the COVID-19 pandemic. Firstly, telemedicine consults should be implemented where possible. Patients can still come to the hospital for their regular blood tests to monitor for any nutritional deficiencies or diabetic control. Subsequent consultations can be performed via telephone or an online platform. Pharmacies should arrange for home delivery services for essential medications. Secondly, online secure electronic platforms can be utilized to conduct group meetings/consults with dieticians and psychologists as required. Online social support groups can also be created. Thirdly, it may be beneficial to initiate a telephone-based intervention to reduce potential weight regains or diabetic relapses. Studies have shown that regular post-operative telephone calls addressing behavior change strategies for diet, physical activity, and nutrition can potentially decrease the incidence of weight regain [22]. Lastly, it should be emphasized to all patients that emergency surgical or medical support will be available at all times should they require any assistance for potential post-operative complications. Postsurgical patients with surgical complications that are lifethreatening or risk permanent organ damage should proceed with surgery as indicated [4]. These include but are not limited to the following: perforated marginal ulcer, bleeding, anastomotic or staple-line leak, intestinal obstruction including internal herniation, gastric band perforation, or prolapse. As surgical teams are being redeployed in alternative roles during this pandemic [23], measures must be taken to ensure the integrity of emergency surgical teams to deal with potential bariatric emergencies.

\section{Preparing for a Post-COVID-19 Era}

The COVID-19 pandemic is expected to be long drawn. However, once the pandemic settles down, measures need to be taken to kickstart weight management and bariatric surgery programs again. The multidisciplinary team will need to prepare for a potential surge in the numbers of patients who will require their services. After a long hiatus, it is also likely that these patients will need to be screened and assessed again prior to surgery. A triaging system is required to decide on how patients should be prioritized to undergo bariatric surgery. We propose that patients who require bariatric surgery for the following reasons are of top priority: revision bariatric surgery for complications (dysphagia, severe reflux disease, malnutrition, anastomotic strictures, slipped band) and patients pending surgery who require pre-operative weight loss (e.g., transplant candidates). The next priority tier should include patients with severe nutritional deficiencies requiring revisional surgery and pre-bariatric surgery patients with poorly controlled comorbidities such as DM, hypertension, hyperlipidemia, osteoarthritis, and obstructive sleep apnea. 


\section{Conclusion}

The COVID-19 pandemic is challenging our world in unprecedented ways. As frontline healthcare workers, it is our priority to join the fight against this novel coronavirus. However, another epidemic, the obesity epidemic, continues to grow. Obesity rates and related metabolic conditions are rising globally over the past few decades [24]. It is vital that we do not neglect this important group of patients.

\section{Compliance with Ethical Standards}

Conflict of Interest The authors declare that there is no conflict of interest.

Human and Animal Rights and Informed Consent This article does not contain any studies with human participants or animals performed by any of the authors. For this type of study, informed consent does not apply.

\section{References}

1. Yeo D, Yeo C, Low TY, et al. Outcomes after metabolic surgery in Asians-a meta-analysis. Obes Surg. 2018;

2. Yeo C, Kaushal S, Lim B, et al. Impact of bariatric surgery on serum uric acid levels and the incidence of gout-a meta-analysis. Obes Rev. 2019;20:1759-70.

3. Brindle M, Gawande A. Managing COVID-19 in surgical systems. Ann Surg 2020.

4. Yang W, Wang C, Shikora S, Kow L. Recommendations for metabolic and bariatric surgery during the COVID-19 pandemic from IFSO. Obes Surg 2020:1-3.

5. Weng TC, Chang CH, Dong $\mathrm{YH}$, et al. Anaemia and related nutrient deficiencies after Roux-en-Y gastric bypass surgery: a systematic review and meta-analysis. BMJ Open. 2015;5(7):e006964.

6. Cotter EW, Kelly NR. Stress-related eating, mindfulness, and obesity. Health Psychol. 2018;37(6):516-25.

7. Rundle AG, Park Y, Herbstman JB, Kinsey EW, Wang YC. COVID-19 related school closings and risk of weight gain among children. Obesity (Silver Spring, Md). 2020

8. Campbell N, Legowski B, Legetic B, et al. Inaugural maximum values for sodium in processed food products in the Americas. $\mathrm{J}$ Clin Hypertens (Greenwich). 2015;17(8):611-3.

9. Dietz W, Santos-Burgoa C. Obesity and its implications for COVID-19 mortality. Obesity (Silver Spring, Md). 2020
10. Muniyappa R, Gubbi S. COVID-19 pandemic, corona viruses, and diabetes mellitus. Am J Physiol Endocrinol Metab. 2020;318: E736-41.

11. Capelo AV, da Fonseca VM, Peixoto MV, et al. Visceral adiposity is associated with cytokines and decrease in lung function in women with persistent asthma. Rev Port Pneumol. 2016;22(5):255-61.

12. Chan AWK, Chair SY, Lee DTF, et al. Tai chi exercise is more effective than brisk walking in reducing cardiovascular disease risk factors among adults with hypertension: a randomised controlled trial. Int J Nurs Stud. 2018;88:44-52.

13. Duggal NA, Niemiro G, Harridge SDR, et al. Can physical activity ameliorate immunosenescence and thereby reduce age-related multi-morbidity? Nat Rev Immunol. 2019;19(9):563-72.

14. Hosseini-Esfahani F, Khalaj A, Valizadeh M, Azizi F, Barzin M, Mirmiran P. Nutrient intake and deficiency of patients 1 year after bariatric surgery: Tehran Obesity Treatment Study (TOTS). J Gastrointest Surg 2020.

15. Kwon Y, Kim HJ, Lo Menzo E, et al. Anemia, iron and vitamin B12 deficiencies after sleeve gastrectomy compared to Roux-en-Y gastric bypass: a meta-analysis. Surg Obes Relat Dis. 2014;10(4):589 97.

16. Coulman KD, MacKichan F, Blazeby JM, et al. Patients' experiences of life after bariatric surgery and follow-up care: a qualitative study. BMJ Open. 2020;10(2):e035013.

17. Jumbe $S$, Meyrick J. Contrasting views of the post-bariatric surgery experience between patients and their practitioners: a qualitative study. Obes Surg. 2018;28(8):2447-56.

18. Aminian A, Vidal J, Salminen P, et al. Late relapse of diabetes after bariatric surgery: not rare, but not a failure. Diabetes Care. 2020;43(3):534-40.

19. Capoccia D, Guida A, Coccia F, et al. Weight regain and diabetes evolution after sleeve gastrectomy: a cohort study with over 5 years of follow-up. Obes Surg. 2020;30(3):1046-51.

20. Sockalingam S, Leung SE, Cassin SE. The impact of COVID-19 on bariatric surgery: re-defining psychosocial care. Obesity (Silver Spring, Md). 2020.

21. Yeo D, Toh A, Yeo C, et al. The impact of impulsivity on weight loss after bariatric surgery: a systematic review. Eating and weight disorders : EWD. 2020.

22. Voils CI, Adler R, Strawbridge E, et al. Early-phase study of a telephone-based intervention to reduce weight regain among bariatric surgery patients. Health Psychol. 2020;39:391.

23. Yeo D, Yeo C, Kaushal S, Tan G. COVID-19 \& the general surgical department - measures to reduce spread of SARS-COV-2 among surgeons. Ann Surg 2020.

24. Mitchell NS, Catenacci VA, Wyatt HR, et al. Obesity: overview of an epidemic. Psychiatr Clin North Am. 2011;34(4):717-32.

Publisher's Note Springer Nature remains neutral with regard to jurisdictional claims in published maps and institutional affiliations. 